\title{
Development of a Heater Structure Based on ITO Films Deposited by RF Magnetron Sputtering for Chip-scale Atomic Clock
}

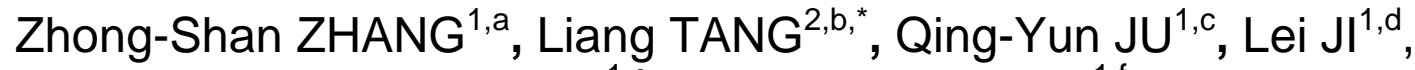 \\ Meng-Hui ZHII,e and Dong-Hai QIAO ${ }^{1, \mathrm{f}}$ \\ ${ }^{1}$ School of Electronic and Information Engineering, Soochow University, Suzhou, Jiangsu, China \\ 2 Institute of Acoustics, Chinese Academy of Sciences, Beijing, China \\ a zhangzhongshan@mail.iggcas.ac.cn, ${ }^{\mathrm{b}}$ tangliang@mail.ioa.ac.cn (corresponding \\ author), ${ }^{c}$ cljqy@126.com, ${ }^{d}$ jileisuda@163.com, ${ }^{e}$ zhimenghui@yeah.net, ${ }^{f}$ qiaodh@suda.edu.cn
}

Keywords: Lndium tin oxide, Physics package, Chip-scale atomic clock, Heater structure

\begin{abstract}
The heater structure plays an important role in the chip-scale atomic clock (CSAC) to achieve the performance of low power consumption, and it is the most effective way to make the structure by depositing the indium tin oxide (ITO) for its heating performance and transparent property. The influence of the factors, including the RF power and the depositing pressure in the fabrication process, on the characteristics of the ITO film is investigated first in this paper. Then the key properties of the ITO film such as the depositing rate, the resistivity, the sheet resistance and the transmittance are tested. The depositing rate is tested by an AlphaStep D-100, the resistivity and the sheet resistance are measured using a four-probe method, and the transmittance is analyzed with a spectrophotometer U-4100. Based on the properties tested above, the optimized process parameters with an RF power of $70 \mathrm{~W}$, a substrate under room temperature and a depositing pressure of $0.2 \mathrm{~Pa}$ are chosen to fabricate the heater structure. Finally, a heater structure with $60 \mathrm{ohm}$ resistance is designed and fabricated. Test results show that the heater structure can heat the physics package of the CSAC up to $85{ }^{\circ} \mathrm{C}$ in less than 420 seconds, which is suitable to be used in the CSACs.
\end{abstract}

\section{Introduction}

CSACs possess advantages of low power consumption and small size, which are good candidates to be applied in the fields of geophysical survey, military equipments and global positioning and navigation. The CSAC is composed of three parts including a physics package, a control loop and an RF module, and the vapor cell inside the physics package is usually heated to about $70-90{ }^{\circ} \mathrm{C}$ to achieve a good performance. There are several ways to make the heater structure inside the physics package for CSACs. ITO films deposited on both sides of the vapor cell can act as a heater structure [1]. Pt films deposited on the vapor cell can be used as a hearter and a thermistor at the same time [2]. The AlSi resistive heater is realized on one side of the vapor cell and spearated by a thin $\mathrm{SiO}_{2}$ dielectric layer [3], and a MOSFET can also be used to act as a heater. In this paper, ITO films deposited by an RF magnetron sputtering method are used to form the heater structure for the heating performance and transparent property. Although there are different methods for ITO deposition including reactive thermal evaporation [4], chemical vapor deposition(CVD) [5], ion beam sputtering [6], and so on, the RF magnetron sputtering method is selected for a high depositing rate, a large depositing area, less damaged areas, better uniformity and better adhesion to the glass substrate comparing with other depositing methods [7,8]. The influence of the factors, including the RF power and the depositing pressure, on the characteristics of the ITO film is investigated first. Then the key properties of the ITO film such as the depositing rate, the resistivity, the sheet resistance and the transmittance are tested. Finally, a heater structure with a Pyrex 7740 glass substrate, a transparent ITO thin film and Au leadwires is designed and fabricated. 


\section{Optimization of the Process Parameters for the ITO Film}

\section{Influence of the RF Power on the Optical and Electrical Characteristics.}

Experiments start with Pyrex 7740 glass substrates with the thickness of 0.3um and the diameter of $50.8 \mathrm{~mm}$. Before the depositing process, all the substrates are ultrasonic cleaned by the acetone and alcohol. Then the substrates are rinsed by the deionized water and dried with the nitrogen blow. The target is a ceramic disc with $10 \% \mathrm{SnO}_{2}$ and $90 \% \mathrm{In}_{2} \mathrm{O}_{3}$ by weight. The base pressure is evacuated to $8 \times 10^{-4} \mathrm{~Pa}$, and then the Ar gas with the high purity of $99.999 \%$ is introduced. The ceramic target is pre-sputtered for $1 \mathrm{~min}$ to remove the impurity. The working pressure is set to $0.2 \mathrm{~Pa}$, and the Ar gas flow is set to $80 \mathrm{sccm}$. The photoresist is used as the sacrificial material to form the structure in the lift-off process, and the temperature of the substrate is set to room temperature. The sputtering time is set to $90 \mathrm{~min}$, and the target-substrate distance is fixed to $60 \mathrm{~mm}$. The RF power is set from $40 \mathrm{~W}$ to $80 \mathrm{~W}$, and the influence of the RF power on the optical and electrical characteristics of the ITO film is shown in Fig.1.

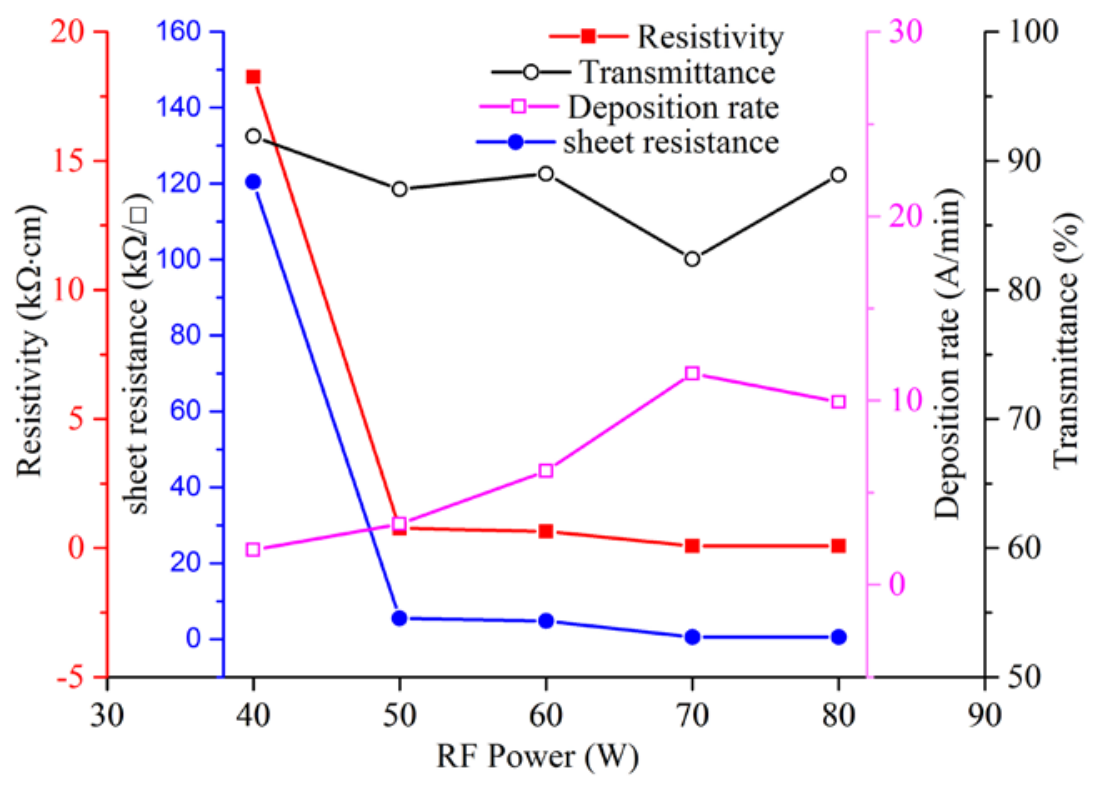

Fig. 1 The influence of the RF power on the optical and electrical characteristics

The results show that the resistivity and the sheet resistance are decreasing as the RF power increases. As can be explained in theory, the increase of the RF power generates more Ar ions. Then more $\mathrm{Sn}^{4+}$ and $\mathrm{In}^{3+}$ metal atoms are sputtered out with high energy onto the substrate. It results in more oxygen deficiency and creates more carrier density, and then decreases the resistivity [9]. The results also show that the ITO film prepared at the RF power of $40 \mathrm{~W}$ has the highest transmittance while the one prepared at the RF power of 70W has the lowest transmittance, which agrees with the change trend of the depositing rate. This phenomenon is due to the grain size. The grain size increases as the thickness of the film increases, which cause the light scattering [10]. When the RF power is in the range of 40-50W, the depositing rate is rather small. As the RF power increases from 50 to $80 \mathrm{~W}$, the depositing rate increases first and then drops, reaching a maximum when the RF power is $70 \mathrm{~W}$. It can be explained as follows. When the film grows initially, the atoms sputtered reach the surface of the glass substrate randomly and there is almost no diffusion along the glass surface due to the limited mobility. It's in a longitudinal growth mode and the nucleation particle is small. As the RF power increases, the atoms sputtered become more energetic and the amount increases at the same time. The atoms reaching 
the surface of the substrate have higher mobility and the lateral growth happens. The increase of the RF power trends to reduce the density of the defects and promotes the growth of the film [11]. However, when the RF power increases to a high level, the atoms sputtered have excessive energy and bombard the surface of the ITO film deposited, causing the decrease of the depositing rate. Based on the results above, the RF power of 70W is chosen as a optimized parameter for the depositing of the ITO film.

\section{Influence of the Working Pressure on the Optical and Electrical Characteristics.}

The RF power is fixed to $70 \mathrm{~W}$, and the rest parameters except the working pressure are the same as that mentioned above. The working pressure is set from $0.2 \mathrm{~Pa}$ to $1.0 \mathrm{~Pa}$, and the influence on the optical and electrical characteristics of the ITO film is shown in Fig.2.

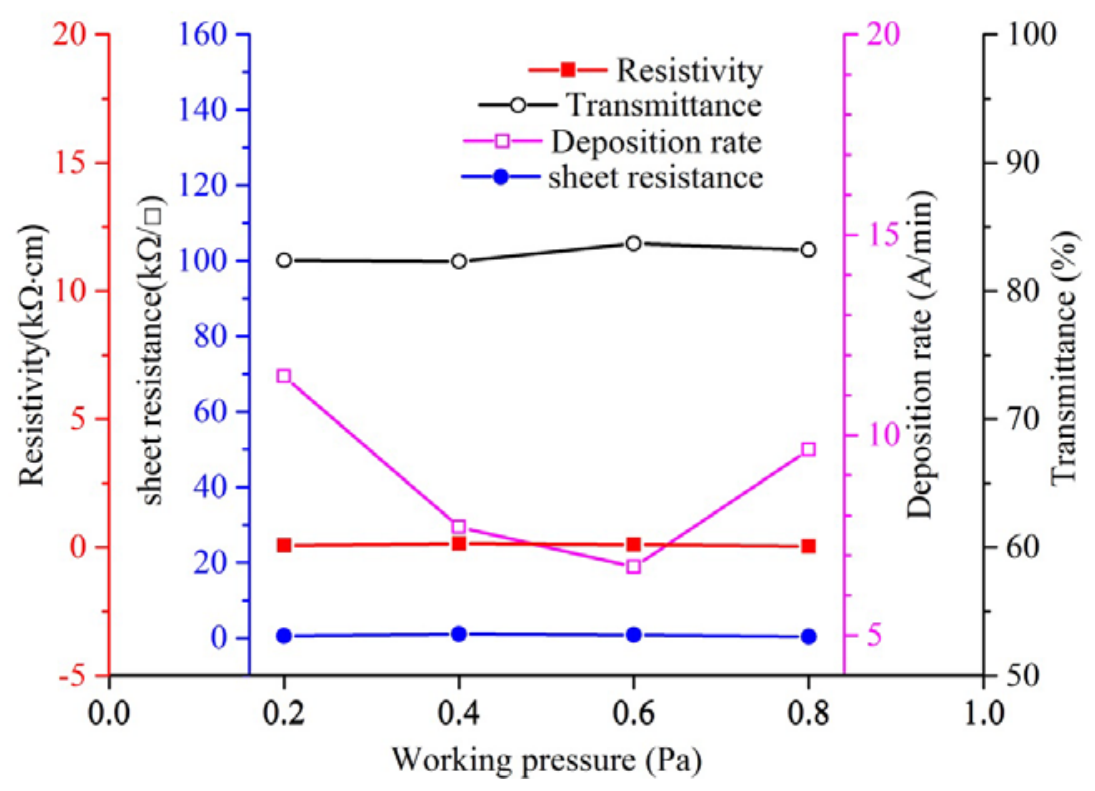

Fig. 2 The influence of the working pressure on the optical and electrical

The results show that the resistivity, the sheet resistance and the transmittance have little changes with the variation of the working pressure, while the depositing rate has significant changes. It can be explained as follows. When the woking pressure is low, most of the ITO molecules can reach the glass substrate because the ITO molecules have a long mean free path, causing the high depositing rate at the low working pressure. As the working pressure increases, the mean free path of the ITO molecules gets shortened, and most of the ITO molecules can't reach the glass substrate, causing the depositing rate to be low. When the working pressure is $0.8 \mathrm{~Pa}$, although the mean free path of the ITO molecules is short, the ITO molecules are sputtered out more. Therefore, the depositing rate increases again. Based on the results mentioned above, the working pressure of $0.2 \mathrm{~Pa}$ is chosen as a optimized parameter for the depositing of the ITO film.

\section{Development and Test of the Heater Structure}

In order to heat the vapor cell inside the physics package of the CSAC to about $85^{\circ} \mathrm{C}$, the resistance of the heater structure could not be too large; otherwise the heating power would be not enough when the heater structure is biased under a constant voltage. The resistance of the heater structure is determined by the resistance of the ITO film with the resistance of the gold wires together. The resistance of the gold wires can be calculated and determined by the length, the width and the thickness of the gold 
wires, which can be controlled precisely. Based on the optimized process parameters, the resistance of the ITO film can be controlled by the sputtering time. The photograph of the heater structure is shown in Fig.3. The fabrication process begins with a Pyrex 7740 glass with the thickness of 500um. Before the depositing process, the substrate is ultrasonic cleaned by the acetone and the alcohol. Then the substrate is rinsed by the deionized water and dried with a nitrogen blow. $\mathrm{Cr}$ and Au films are sputtered on the substrate and patterned by the photolithograph to form the gold wires. The ITO film deposited by the optimized parameters is patterned using the lift-off method. Table 1 shows the process parameters used in the fabrication. The heater structure is heated inside a seal chamber, and Fig. 4 shows the temperature vs. the heating time. The temperature of $85^{\circ} \mathrm{C}$ can be achieved in about 420 seconds.

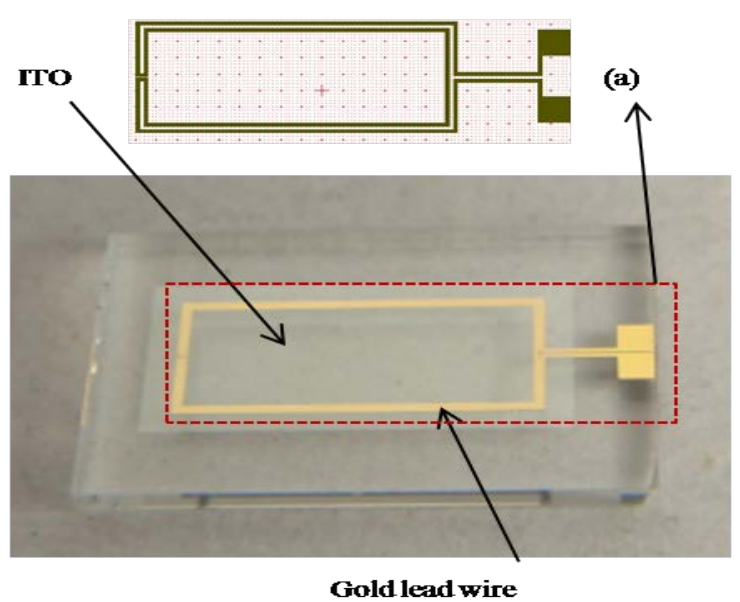

Fig. 3 Photograph of the heater structure

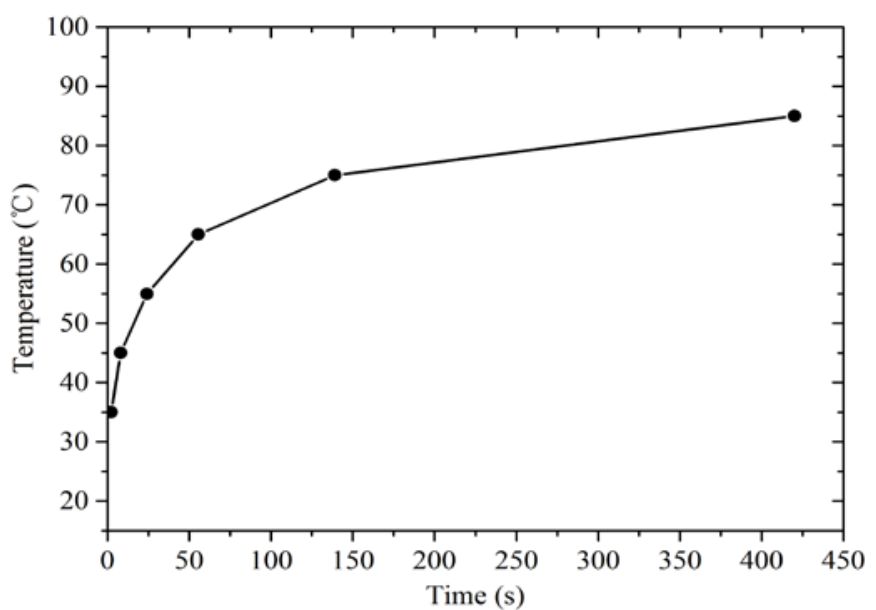

Fig. 4 Temperature vs. heating time by the heater

Table 1 The Process parameters used in the fabrication of the heater structure

\begin{tabular}{llll}
\hline Material & $\mathrm{Cr}$ & $\mathrm{Au}$ & $\mathrm{ITO}$ \\
\hline Base pressure[Pa] & $8.4 \mathrm{E}-4$ & $8.4 \mathrm{E}-4$ & $7.4 \mathrm{E}-4$ \\
Sputtering pressure[Pa] & 0.3 & 0.3 & 0.2 \\
Gas/Flow rate[sccm] & $\mathrm{Ar} / 80$ & $\mathrm{Ar} / 80$ & $\mathrm{Ar} / 80$ \\
RF power[W] & 100 & 100 & 70 \\
Bias power[V] & 120 & 140 & 120 \\
Time[min] & 20 & 25 & 30 \\
Target & Cr target & Au target & ITO target \\
& & & \\
\hline
\end{tabular}

\section{Summary}

The heater structure plays an important role in the CSAC to achieve the performance of low power consumption, and it is the most effective way to make the structure by depositing the ITO film for its heating performance and transparent property. The influence of the factors, including the RF power and the depositing pressure in the fabrication process, on the characteristics of the ITO film is investigated first in this paper. Then the key properties of the ITO film such as the depositing rate, the resistivity, the sheet resistance and the transmittance are tested. Based on the properties tested, the optimized process parameters with an RF power of $70 \mathrm{~W}$, a substrate under room temperature and a depositing pressure of $0.2 \mathrm{~Pa}$ are chosen to fabricate the heater structure. Finally, a heater structure with $60 \mathrm{ohm}$ 
resistance is designed and fabricated. Test results show that the heater structure can heat the physics package of the CSAC up to $85^{\circ} \mathrm{C}$ in less than 420 seconds, which is suitable to be used in the CSACs.

\section{Acknowledgement}

This work is supported by Youth Innovation Promotion Association CAS, the National Natural Science Foundation of China (Grant No.11104313) and Sinoprobe-09-02-03.

\section{References}

[1] S. Knappe, P. Schwindt, V. Shah, L. Hollberg, J. Kitching, L. Liew, J. Moreland, Microfabricated Atomic Frequency References, IEEE International Ultrasonics, Ferroelectrics, and Frequency Control Joint 50 ${ }^{\text {th }}$ Anniversary Conference. (2004)87-91.

[2] S. L. Li, J. Xu, Z. Q. Zhang, L. B. Zhao, L. Long, Y. M. Wu, Integrated Physics Package of a Chip-Scale Atomic Clock, Chinese Physics. B. 23(2014)074302-1-5.

[3] T. Overatolz, J. Haseler, G. Bergonzi, A. Pezous, P. A. Clerc, S. Ischer, J. Kaufmann, M. Despant, Wafer Scale Fabrication of Highly Integrated Rubidium Vapor Cells, The $27^{\text {th }}$ International Conference on Micro Electro Mechanical System. (2014)552-555.

[4] J. Du, X. L. Chen, C. C. Liu, J. Ni, G. F. Hou, Y. Zhao, X. D. Zhang, High transparent and conductive indium tin oxide thin films for solar cells grown by reactive thermal evaporation at low temperature, Applied Physics A: Materials Science and Processing. 112(2)(2014)815-822.

[5] Ikenoue, Takumi, Sakamoto, Shin-ichi, Inui, Yoshitaka, Fabrication and characterization of Cu2O, $\mathrm{ZnO}$ and ITO thin films toward oxide thin film solar cell by mist chemical vapor deposition method, Physica Status Solidi (C) Current Topics in Solid State Physics. 11(7-8)(2014)1237-1239.

[6] W. J. Geerts, N. A. Simpson, A. D. Woodall, M. S. Compton, Indium tin oxide thin films deposited at low temperature using dual ion beam sputtering, Materials Research Society Symposium Proceedings. 1699(2014).

[7] U. Betz, M. Kharrazi Olsson, J. Marthy, M. F. Escolá, F. Atamny, Thin Films Engineering of Indium Tin Oxide: Large Area Flat Panel Displays Application, Surface and Coatings Technology. 200(2006)5751-5759.

[8] Y. Navid, B. Behrang, Y. Payam, R. Bizhan, S. Pezhman, Optimization of Sputtering Parameters for the Deposition of Low Resistivity Indium Tin Oxide Thin Films, Acta Metallurgica Sinica. 27(2014)324-330.

[9] G. S. Zhu, Z. P. Yang, Effect of Sputtering Power and Annealing Temperature on the Properties of Indium Tin Oxide Thin Films Prepared from Radio Frequency Sputtering Using Power Target, Journal of Materials Science: Materials in Electronics. 24(2013) 3646-3651.

[10]A. Eshaghi, M. Pakshir, R. Mozaffarinia, Preparation and Photo-Induced Super-Hydrophilicity of Composite $\mathrm{TiO}_{2}-\mathrm{SiO}_{2}-\mathrm{In}_{2} \mathrm{O}_{3}$ Thin Film, Applied Surface. Science. 256(2010)7062-7066.

[11]Y. P. Chao, W. Tang, X. H. Wang, Properties of Resistivity, Reflection and Absorption Related to Structure of ITO Films, Journal of Materials Science Technology. 28(2012)325-328. 